\title{
ANALISI CONTRASTIVA ITALIANO-CROATO-CECA NELLA FRASEOLOGIA ENTOMOLOGICA
}

Irena Marković, Sveučilište u Zadru, imarkov@unizd.hr

Monika Berić, moberic93@gmail.com

10.31902/fIl.30.2020.14

UDK 811.131.1'373.61

Nella presente ricerca si è andati ad investigare in modo contrastivo quanto il comportamento e le caratteristiche degli insetti (in generale considerati universali) differiscano nelle tre lingue (italiano, croato e ceco) nelle espressioni idiomatiche.

Si è partiti dalle espressioni idiomatiche italiane contenenti una componente entomologica (17 insetti), le quali cercano i loro equivalenti nel croato e nel ceco. Significa che l'analisi viene fatta soltanto unidirezionalmente dall'italiano al croato e ceco.

Da un lato, la lingua croata e quella ceca, essendo della stessa origine (slava), dovrebbero accomunare equivalenti fraseologici totali (attraverso la variante protoslava), oppure attraverso i prestiti di terminologie religiose, ad esempio bibliche. D'altra parte, per via del diretto contatto italiano-croato, il croato dovrebbe avere più similarità fraseologica con l'italiano, a differenza del ceco. In base ai 4 passi della linguistica contrastiva e selezionando gli equivalenti totali, parziali e quelli diversi ci si è concentrati a spiegare (anche attraverso un'analisi concettuale) il perché delle diversità e quali siano le scelte linguistiche nei diversi equivalenti.

Delle 72 espressioni in italiano, soltanto 9 (il 12\%) sono equivalenti totali in tutte e tre le lingue. Differenziando le lingue, si nota un'affiliazione più stretta tra le lingue slave (24 equivalenze totali), che non tra il croato e l'italiano (15) e tra il ceco e l'italiano (11). L'analisi concettuale conferma questa affiliazione slava.

Le lingue slave (croato e ceco), anche se storicamente e geograficamente più lontane, hanno conservato le loro specificità idiomatiche. D'altra parte l'italiano, essendo nell'ultimo millennio in stretto rapporto con il croato, ha sicuramente lasciato più repertorio idiomatico nel croato che nel ceco.

Parole chiave: espressioni idiomatiche, analisi contrastiva, analisi concettuale, insetti, equivalenti, italiano, croato, ceco. 


\section{Introduzione}

Diversi sono gli studi condotti sugli animali (domestici o selvatici) nella fraseologia croata, spesso sottoposta ad un'analisi contrastiva anche con altre lingue (polacco, ceco, russo, lingue romanze, lingue germaniche ${ }^{1}$. Nonostante ciò, nel presente studio ci si è concentrati e ci si è limitati alla classe degli insetti, per approfondirne l'analisi qualitativa. Questa classe rappresenta la più grande classe tra i vari raggruppamenti dell'intero regno animale. Gli insetti, come la maggior parte del regno animale, esistevano prima dell'esistenza umana e la vita dell'uomo è sempre e continuamente stata in stretta relazione con gli insetti. Ciò fa sì che l'uomo cominci a produrre significati basandosi sull'aspetto o sul comportamento degli animali, attribuendoli anche all'uomo (zoomorfismo); oppure comincia ad attribuire caratteristiche umane agli animali (antropomorfismo), ancora una volta per ottenere significati diversi. Mentalmente, la prima associazione nel menzionare gli insetti è la loro dannosità (cimice, pidocchio, zanzara). Nonostante ciò, e anche se spesso accettiamo tale connotazione negativa come la più frequente delle espressioni con gli animali in generale (Vidović Bolt 2), sappiamo che queste connotazioni negative possono essere anche ambivalenti (possono avere connotazioni positive a seconda del tono, dello stato emotivo dell'interlocutore o del contesto). Dall'altro lato, si contrappone l'utilità di altre specie di insetti come l'ape, la farfalla, la formica. Analizzando in che misura un determinato popolo trasferisca tratti degli insetti all'uomo o agli oggetti, descrivendo così l'uomo o la situazione come positiva o negativa, si può vedere l'aspetto della cultura di un popolo. Inoltre, I'analisi contrastiva riguarda anche lo sfondo geografico. Non si può usare un determinato insetto se non vive nel suo ambiente naturale, o, quando lo si fa, sappiamo che si sta parlando di un prestito o di un calco.

${ }^{1}$ La maggior parte di questi lavori si trova in Životinje $u$ frazeološkom ruhu (2014). Atti del convegno di Zagabria tenutosi nel 2014, che accomuna diverse ricerche del campo animalesco comparato e contrastato con diverse lingue (polacco, russo, ceco, lingue romanze, germaniche ecc.). In seguito Barčot (2014) con il croato, il russo e il tedesco; Bunk e Opašić (2010) con il croato e il ceco. Per i prestiti dei termini religiosi (es. biblici) vedi Jelaska (2014). Per i contatti italiano-croati nella fraseologia vedi Jernej (1996) e Turk $(1994,2014)$. Per la motivazione nell'interpretazione delle espressioni idiomatiche vedi Marković (2018). Per altri lavori correlati al tema vedi la tesi di laurea di Delić, M. (2014) Animali nella fraseologia italiana ed equivalenze francesi e croate, Jerolimov, I. (2001) Frazemi sa somatskom sastavnicom na primjeru talijanskohrvatske frazeologije, Menac-Mihalić, M. (2000) Frazemi s nazivima kukaca u hrvatskim govorima. 


\section{Metodologia della ricerca}

Tenendo presente i quattro passi essenziali dell'analisi contrastiva, nella parte selettiva abbiamo analizzato tre lingue (italianocroato-ceco), proseguendo unidirezionalmente dalla lingua italiana a quella croata e poi a quella ceca. Generalmente abbiamo usato anche la bidirezionalità per confermare le diverse equivalenze (equiv.) in croato e in ceco, che hanno di ritorno un equivalente uguale in italiano. Servendosi di diversi dizionari dei modi di dire, prima di tutto in italiano ${ }^{2}$, si è cercato di accumulare esaurientemente un numero totale di espressioni idiomatiche contenenti una componente di insetto e si è arrivati a 72 espressioni idiomatiche. Per la parte croata sono stati consultati dizionari ${ }^{3}$, molto meno esaurienti dei dizionari italiani, il che ci ha portato al bisogno di generare nuove fonti sui modi di dire che non esistono in nessuno dei dizionari, ma hanno trovato conferma nei libri o nei giornali croati (specificamente notificati nell'analisi). Per la lingua ceca sono stati consultati dizionari specializzati ${ }^{4}$, mentre per tutte e tre le lingue sono stati trovati dei dizionari bilingui che sono stati soltanto parzialmente d'aiuto, perché non completi e non esaurienti come i dizionari monolingui. ${ }^{5}$ La corrispondenza sia strutturale che contestuale non viene sempre rispettata. Quando non ci sono

${ }^{2}$ Dizionari monolingui: Vocabolario della lingua italiana, Lo Zingarelli 1996, Dizionario dei modi di dire di Monica Quartu, Grande dizionario Hoepli italiano di Gabrielli Aldo, Metafore ed espressioni idiomatiche di Casadei (1996), Dizionario della lingua italiana sulle diverse pagine web (grandidizionari.it e dizionari.repubblica.it) e Vocabolario on line Treccani e Sapere.it.

${ }^{3}$ Dizionari monolingui: Hrvatski frazeološki rječnik (Menac e altri), Hrvatski jezični portal, Baza frazema hrvatskog jezika, Veliki rječnik hrvatskog jezika (Anić).

4 Dizionari monolingui: dizionari online come Slovník spisovného jazyka českého di B. Havránka, J. Běliče, M. Helcla a A. Jedličky e Slovník české frazeologie a idiomatiky (rozdělený na část slovesnou a neslovesnou) di DEBDict presso l'Università di Masaryk nella Repubblica Ceca, Encyklopedický slovník češtiny di P. Karlik, M. Nekula, J. Pleskalova (ed.).

Un grande aiuto per verificare e correggere i modi di dire in ceco è stata la professoressa Kristýna Rygolová, Università di Zara.

${ }^{5}$ Vocabolario italiano-croato (2012) e Hrvatsko-talijanski rječnik (1994) di Jernej e Deanović, Hrvatsko-talijanski frazeološki rječnik di A. Menac e Z. Vučetić, Hrvatsko-romansko-germanski rječnik poredbenih frazema di Ž. F. Arsovski, Hrvatsko-slavenski rječnik poredbenih frazema di Ž. F. Arsovski e Hrvatsko-češko-slovački rječnik di A. Menac, D. Sesar e R. Kuchar, Hrvatskočeški rječnik (1999) di D. Profeta e A. Novosad. 
equivalenti appropriati per un contesto neutrale, vengono presentati anche i modi di dire derogativi o gergali.

Per quanto riguarda il criterio di equivalenza abbiamo selezionato quattro diversi gruppi nell'analisi contrastiva:

1. Equiv. totale: il determinante, cioè l'insetto, uguale alla lingua di partenza, immagine evocata identica, stessa struttura della proposizione con verbi, complementi, aggettivi, congiunzioni comparative, ecc...; anche con possibili tenui modificazioni, significato completamente identico. Per esempio: operoso come un'ape/ marljiv kao pčela/ pilný jako včela.

2. Equiv. parziale: si distingue soltanto nella struttura della proposizione (cambiamento nell'ordine delle parole nella frase, verbi diversi o sinonimi, con o senza una congiunzione comparativa, con omissione o aggiunta di parole), come gli aggettivi, ma sempre con il determinante conservato, l'immagine evocata e il significato mantenuti parzialmente o identici. Per esempio: (numerosi) come formiche/ kao mrava <koga>/ je někoho <tam> jako mravenců. ${ }^{6}$

3. Disuguaglianza totale: esiste quando i modi di dire non hanno lo stesso determinante (non usano insetti o animali in generale), l'immagine evocata è completamente diversa, la forma della proposizione non è uguale, ma il significato viene mantenuto. Per esempio: in bocca chiusa non entrano mosche/ od mučanja (šutnje) glava ne boli/ mlčeti zlato, mluviti střibro.

4. Senza equivalente: accomuna tutti i modi di dire che non hanno un equiv. adeguato né in croato né in ceco.

L'obiettivo di questo studio non era quello di redigere un vocabolario, ma piuttosto di creare un documento di ricerca tramite un'analisi contrastiva trasparente e soggetta a modifiche. Un corpus di

\footnotetext{
${ }^{6}$ In questo gruppo abbiamo permesso due eccezioni: avere grilli per la testa/ imati mušice (muhe, bube, bubice) u glavi/ mít mouchy (brouky) v hlavě. Se guardiamo attentamente soltanto il determinante non è uguale, ma nella classificazione si tratta di insetti dello stesso tipo (grillo-moscerino). La struttura della proposizione, l'immagine evocata e il significato sono uguali. II secondo esempio è: aggirarsi (essere) come una mosca senza capo/ chodit (běžet) jako bezhlavý (bez hlavy). Il determinante mosca manca nel modo di dire ceco, però gli altri parametri corrispondono.
} 
questi modi di dire può servire da sfondo per un dizionario. Nulla è stato incluso senza prima essere stato verificato nei dizionari, nei dizionari online e in altre fonti bibliografiche.

\section{Analisi contrastiva dei modi di dire}

Nel confronto vengono analizzati in italiano 17 insetti: ape, calabrone, cavalletta, cicala, cimice, farfalla, formica, grillo, lucciola, mosca, moscerino, piattola, pidocchio, pulce, tignola, vespa e zanzara, per un totale di 72 modi di dire. Cercando questi 72 modi di dire nel repertorio idiomatico croato, si nota che gli insetti sono presenti nella lingua croata soltanto in 34 modi di dire, con alcuni insetti, come per esempio la mosca che accomuna un unico significato l' 'essere fastidiosi' presentato da diversi insetti in italiano (zanzara, vespa, pulce, calabrone) e con due insetti non menzionati nella fraseologia italiana (trut 'fuco' e buba 'scarafaggio'). Nella lingua ceca, invece, vengono menzionati 13 insetti, con 28 espressioni idiomatiche. La maggioranza delle espressioni idiomatiche italiane, precisamente 20 esempi, appartiene al determinante $\operatorname{mosca}^{7}$ (quasi il 30\%), alquanto numerosi in croato e in ceco. Segue il grillo con dieci modi di dire, tradotto in croato come zrikavac. Nella lingua ceca esiste un nome comune per questo insetto curček (è stato notato un numero bassissimo di specie di Gryllidae sul terreno geografico ${ }^{8}$ ) (Holuša et all. 2007). Nella lingua ceca esistono alcune espressioni idiomatiche con l'insetto cvrček come: nasadit někomu curčka do hlavy, být malý jako cvrček, però esse non corrispondono agli equivalenti italiani. Non sorprende allora che in ceco, ma anche in croato, non sia stato trovato neanche un modo di dire relativo a questo insetto se si parte dalle espressioni idiomatiche italiane. In Italia, invece, i grilli hanno una forte incidenza nell'immaginario popolare, dalla Festa del grillo, ai modi di dire indovinala grillo, ai personaggi fiabeschi, il Grillo Parlante, al comico Beppe Grillo che ha giocato sul suo cognome indicante chi si prodiga a dare consigli rimanendo inascoltato. Interessante che in croato i grilli, pur presenti, non vengano assolutamente menzionati nelle espressioni. Dopo la mosca ed il grillo, il determinante usato più frequentemente è la formica, presentato dai nove modi di dire italiani (in croato mrav, altrettanto numerosi, mentre in ceco mravenec 6 ). Gli altri insetti come la pulce (in croato buha 4 espressioni; in ceco blecha 2) vengono

\footnotetext{
${ }^{7}$ Interessante la parola it. mosca, cr. muha, cec. moucha hanno probabilmente la stessa origine indoeuropea $\leftarrow i e .{ }^{*}$ mows-/mus- (lat. musca, grč. myîa).

${ }^{8}$ I grilli sono notissimi per aver abitato tutta la terra eccetto i terreni della latitudine a nord e a sud del $55^{\circ}$ parallelo.
} 
menzionati in 5 modi di dire italiani. Gli altri insetti si trovano in pochi modi di dire come la vespa (osa-vosa), in quattro modi di dire in contrasto con il croato che ne rimane senza e il ceco con 2 modi di dire; la cicala (cvrčak-cvrček) non presente né in ceco né in croato, la farfalla (leptir-motýl) con un modo di dire in croato altrettanto come in ceco, il pidocchio (uš-veš) con una espressione in croato e in ceco. La zanzara (komarac-komár) in tre espressioni, altrettanto come in croato, e in ceco 2, l'ape (cr. pčela e cec. včela) in una espressione, il calabrone (stršljen-sršeň) non presente in croato e in ceco, la cavalletta (cr. skakavac con una espressione, mentre in ceco 3), la lucciola (ivanjska krijesnica-svatojánská muška) in due espressioni, mentre in croato e in ceco non esistono. Esiste soltanto una espressione in italiano con i sostantivi cimice (stjenica-štěnice), moscerino (mušica-muška), piattola (stidna uš-krab) e tignola (moljac-mol), altrettanto in croato e in ceco.

Da tutto il corpus raccolto e analizzato qualitativamente le espressioni idiomatiche possono prima di tutto essere differenziate in base all'associazione positiva o negativa degli insetti. Così per esempio alcuni insetti hanno soltanto connotazioni positive come le api, alcuni sono percepiti sia come positivi che negativi (cicala, farfalla, formica), mentre non sorprende che per la maggioranza degli insetti (nel $90 \%$ circa dei casi) sia sempre presente una connotazione negativa (calabrone, mosca, pidocchio, pulce, zanzara, vespa). Ciò è conforme alle tendenze generali delle espressioni idiomatiche che molto più spesso possiedono connotazioni negative (Vidović Bolt 2014, 1). Un'altra conclusione generale è che tutte le espressioni idiomatiche con significati positivi esistono nella maggior parte dei casi come equivalenti assoluti nelle tre lingue oggetto di ricerca.

\subsection{Analisi concettuale}

Essendo composte dalle metafore tutte le espressioni idiomatiche possono accomunare diversi concetti di caratteristiche psicologiche dell'uomo e i suoi difetti. Nella stessa maniera possono anche descrivere le caratteristiche fisiche dell'uomo e le situazioni che ci circondano. Le motivazioni che spingono l'uomo a collegare un ente ai significati diversi possono essere varie. Per esempio, Vidović Bolt (418) differenzia motivazione concreta (risultato dell'esperienza con l'animale - ammassare/accumulare come una formica), motivazione associativa (conseguenza di una percezione abituale/stereotipizzata del comportamento dell'animale - girare come una mosca senza capo), motivazioni illogiche $o$ assurde (non hanno alcuna spiegazione logica non cavare un grillo dal buco), motivazioni non reali (che non sono possibili - essere una mosca bianca). Inoltre, anche se concettualmente 
diverse, le espressioni idiomatiche possono essere motivate dalla Bibbia (laborioso come una formica) o essere culturo-specifiche o nazionali (/ mirna Bosna).

1. Le caratteristiche fisiche sono direttamente legate a motivazioni concrete e reali e si notano in particolare in tre espressioni: essere nero come un calabrone, avere un vitino di vespa, magro come un moscerino. La prima in italiano ha anche un significato di stato psichico di rabbia, mentre in croato e in ceco le varianti non presentano un insetto (cr. biti crn kao Ciganin, cec. černý jako cikán) e rappresentano soltanto il colorito o la sporcizia della pelle. L'immagine mentale che sta dietro al vitino di vespa è assolutamente trasparente, però non conosciuta alla lingua croata che usa un altro animale cr. vitka kao srna, anche se nella lingua ceca combacia perfettamente con l'espressione italiana. La persona che è magra come un moscerino ha sicuramente lo stesso livello di comparazione della zanzara che sta dietro l'immagine mentale dell'espressione croata e ceca (cr. suh kao komarac, mršav kao glista e cec. slabý (hubený) jako komár, být hubený jako žížala).

2. Caratteristiche psicologiche:

a) Bontà vs. cattiveria - Nelle espressioni idiomatiche sia italiane che slave si possono trovare insetti per esprimere sia la bontà che la cattiveria dell'uomo. Così con non far male a una mosca si esprime lo stesso concetto in croato, che utilizza, però, l'animale formica (cr. ni mrava ne bi zgazio), mentre il ceco ha più corrispondenza con la variante italiana 'non ucciderebbe neanche una mosca' (altrettanto conosciuta con il pulcino). La cattiveria, invece, viene espressa da due insetti in italiano. L'espressione pungere come una vespa non riguarda soltanto l'atto del parlare, ma porta con sé una connotazione particolare, ossia quella di dire le cose intenzionalmente in modo brusco. Gli equivalenti croato e ceco non presentano un insetto nelle loro espressioni, anche se l'animale (serpente) è quello più vicino all'immaginario comune (cr. imati zmijski jezik/oštar jezik e cec. mít ostrý jazyk/mít jazyk jako břitva/mít jazyk z hadích ocásků (spletený)).

b) Stupidità vs. intelligenza - Analizzando le diverse espressioni idiomatiche nelle quali è presente un significato di stupidità (capacità mentali ridotte) si nota che gli insetti non sono molto suggestivi nel motivare questo significato.

In italiano l'espressione avere il cervello di una 
formica/grillo è l'unica espressione con il significato nominato (proprio per la piccolezza dell'insetto/cervello), mentre gli altri animali sembrano essere molto più adatti allo stereotipo cercato (gallina, asino, oca, cavallo). Così in croato ed in ceco abbiamo espressioni che combaciano assolutamente prendendo in considerazione la gallina o l'uccello (cr. pileći/kokošji/ptičji mozak e cec. mít slepičí rozum).

c) Pensieri e comportamenti improvvisi e illogici - I comportamenti e $\mathrm{i}$ pensieri che non vengono percepiti come 'normali' vengono espressi similmente nella lingua italiana e nelle due lingue slave, usando in tutti e tre i casi un insetto come determinante it. avere grilli per la testa (imati mušice/bube/bubice 'mosche, scarafaggi' u glavi sia in croato che in ceco), ma sempre con un'immagine evocata molto simile. Mentre nell'espressione gli è venuto un grillo 'capriccio, voglia bizzarra', il ceco usa il determinante 'mosca', e nel croato non viene menzionato alcun animale (šunuti u glavu). Per la terza espressione aggirarsi come una mosca senza capo, Turk e Spicijarić $(2014,10)$ suppongono un influsso della lingua italiana su quella croata (equiv. totali), proprio perché nel ceco manca il determinante 'mosca' ('correre senza capo').

d) Presunzione vs. umiltà - La particolarità di questa espressione pidocchio rifatto (ripulito) è che soltanto l'italiano usa il pidocchio per l'immagine mentale di una persona grezza e volgare che si è arricchita, mentre nelle due lingue slave non esiste. Un'altra espressione accomuna un simile significato fare la mosca cocchiera, una persona che non soltanto è fastidiosa e insopportabile, ma pretende di essere il capo e si assume da solo compiti di responsabilità. Tutte e due le espressioni non hanno un equiv. né formale né semantico totale. II significato (in una parte) viene invece mantenuto attraverso equiv. totali con altri animali ponašati se kao puran 'pavone' (in tutte e tre le lingue), motivate dal comportamento di pavoneggiarsi per il cortile.

e) Invadente, fastidioso vs. non invadente, piacevole Sembra che gli insetti siano assolutamente perfetti ad esprimere il concetto di fastidio e invadenza, un universale presente anche nelle nostre tre lingue. Così in italiano troviamo espressioni con diversi insetti noioso come una 
mosca (pulce, zanzara, vespa, calabrone), mentre in croato e in ceco troviamo insetti come mosca, pidocchio, cimice, zecca e in croato specialmente troviamo altri animali verme, verme solitario. Significa che l'espressione con la mosca è l'unico equivalente totale nelle tre lingue. L'espressione essere come le cavallette non è completamente equivalente né nella forma, né nel significato (in croato e in ceco invece lo è), anche se si usa comunque un insetto. In italiano si riferisce ad una persona molto invadente, mentre in croato e in ceco ad un gruppo di persone che invade uno spazio come le cavallette (immagine presa da connotazioni bibliche). Invadenti sono anche le mosche che si devono levare di torno, un'altra espressione che rimane sola e senza un equivalente totale in croato e in ceco (in ceco non esiste un'espressione idiomatica con tale significato), anche se in croato si usa un'espressione con un'immagine mentale simile (cr. riješiti se nametnika 'levarsi i parassiti di torno'). In questa categoria, anche se motivata dall'aspetto fisico dell'insetto (mosca nera), essere una mosca nel latte evoca l'idea di una persona di disturbo che irrita con la propria intromissione. Un'altra espressione che combacia soltanto parzialmente con i significati croati e cechi (bosti u oči - di significato soltanto visuale) che sono equivalenti totali.

f) Parlare vs. tacere - In questa categoria la linea di separazione tra la connotazione positiva e quella negativa è molto vulnerabile, perché la saggezza del tacere nell'espressione in bocca chiusa non entrano mosche può assumere anche un ruolo di rimprovero riferito a coloro che parlano molto. Questo proverbio toscano, assolutamente culturo-specifico, costituisce uno dei principali elogi popolari del Silenzio. La sua specificità non viene trasmessa alle lingue slave, nelle quali l'immagine evocata è differente ed il determinante mosca non esiste (cro. 'il silenzio è oro', ceco 'tacere oro, parlare argento'). In croato troviamo un altro determinante che è legato alla bocca, però non include un insetto, cr. od mučanja glava ne boli. Negli altri due detti, stare zitto come un pidocchio, mettere la pulce in un orecchio, la connotazione negativa è assoluta. Nella prima espressione nella variante italiana non si nota tanto questa connotazione, quanto invece è presente nelle varianti slave; šutjeti kao kurva 'zitta come 
una puttana' nel significato di essere insidiosi, tacere insidiosamente. Secondo Fink Arsovski (46), che ha studiato le espressioni idiomatiche in italiano, esiste una tendenza più eufemistica e questo fa parte della cultura sociale e della mentalità italiana. Per la seconda espressione esiste una equivalenza totale tra italiano e croato (cr. staviti buhu $u$ uho), anche se in croato esiste la variante con lo scarafaggio 'buba', la quale (accanto alla variante mosca) si usa nell'espressione ceca e si mette sul capo e non in un orecchio.

g) Laboriosità vs. pigrizia - Parliamo di nuovo di un campo e di un'immagine mentale ottimale per esprimere e stimare quanto uno lavora. Tra tutti i rappresentanti del mondo animale, gli insetti sono i più presenti in questi modi di dire, motivati da comportamenti concreti. Così in italiano abbiamo operoso come un'ape, laborioso come una formica, con tutte le loro varianti e significati aggiunti 'buona economia, risparmiatore' fare/essere la/una formica, parsimonioso e attivo come una formica, ammassare quanto una formica). Nel caso degli altri animali (mulo, cane, scimmia) si passa quella linea di demarcazione che separa il lavorare (positivo) dal lavorare troppo (negativo). Nelle lingue slave si usano lo stesso tipo di insetti ed espressioni identiche (considerate universali), soltanto l'ammassare come una formica in croato e in ceco trova equivalente parziale con il criceto 'hrčak' e 'hřeček', però di nuovo con un significato più negativo di 'accumulare il non necessario'. Un minimo spostamento di significato lo troviamo nel fare le scarpe alle mosche con il significato di 'essere capace' con equivalenti completamente diversi in croato e in ceco (cr. imati žicu za nešto, cec. jít někomu něco od ruky). Inoltre, altri tipi di insetti rappresentano un'immagine mentale chiara della pigrizia: guardare le mosche che volano, pigliar mosche, andar a caccia di farfalle, fare come la cicala 'spendere in modo poco oculato' e di non concludere nulla non cavar un grillo dal buco, restare con un pugno di mosche. Se paragoniamo tutte queste espressioni (contenenti connotazioni negative) con le lingue slave, non troviamo neanche un equivalente totale o parziale. La lingua italiana in questo campo è estremamente produttiva nell'utilizzare gli insetti per esprimere la poca efficacia dell'uomo. 
Generalmente le motivazioni sono illogiche e non rappresentano un comportamento reale degli insetti.

h) Paura vs. coraggio - Delle tre espressioni analizzate aver paura di una mosca, cuore di grillo, saltare come punto da una vespa soltanto la variante con la mosca ha un equivalente totale con la lingua croata ed esprime il concetto di 'aver molta paura'. Per il ceco l'equivalente sarebbe legato ad un 'cagnolino'. Il coniglio e il cervo, invece, esprimono sia in croato che in ceco lo stesso concetto. Mentre l'immagine mentale del cuore di grillo (in italiano anche cuor di coniglio o essere un coniglio) è presente soltanto nella lingua italiana. La terza espressione saltare come punto da una vespa è specifica sia per la lingua italiana che per quella ceca (vyskočit jako když ho vosa bodne). II suo significato può includere, ma non necessariamente, anche qualcuno che salta improvvisamente dalla paura (un'immagine abbastanza trasparente). In croato esistono espressioni con lo stesso significato in cui non sono presenti la vespa né altri animali (cr. skočiti kao ofuren).

i) Furbizia vs. ingenuità - Anche se molto diversi, i seguenti insetti in italiano vengono usati per esprimere la furbizia o l'ingenuità in diversi gradi (dalla connotazione positiva dell'abilità fino a quella negativa dell'inganno). La motivazione non deriva direttamente dal loro comportamento reale che viene zoomorfizzato in riferimento agli esseri umani. Le espressioni sono specifiche dell'italiano e non trovano alcun equivalente né parziale né totale nelle lingue slave. Così, per ingannare qualcuno si fa la cavalletta a qualcuno (cr. nasamariti/namagarčiti nekoga, cec. hazet klacky pod nohy). Dall'altra parte, girare intorno al lume come le farfalle, con il significato di 'leggerezza e non cautela di qualcuno che è vicino ad un pericolo', non contiene un equivalente nelle lingue slave. Mentre le espressioni dare ad intendere lucciole per lanterne 'far credere una cosa per un'altra, ingannare' e grattare la pancia alla cicala 'motivare qualcuno a confessare/parlare' si trovano anche nelle espressioni slave, però con equivalenti diversi nella forma e nell'immagine mentale. Così in ceco si 'vendono stronzi di topi per pepe', mentre in croato si 'vendono coglioni per reni' o il 'corno per la candela'. La stessa situazione è 
presente in italiano nell'immagine di sfondo in cui, antropomorfizzando l'azione di fare il solletico alla cicala, si può interpretare il significato. In contrasto con l'italiano le lingue slave hanno un equivalente parziale 'forzare qualcuno a cantare', anche se il ceco, come nell'espressione precedente, presenta nuovamente un animale ('forzare l'uccello a cantare').

j) Esagerazione - Gli insetti sembrano essere molto efficaci nel rappresentare azioni o comportamenti esagerati degli uomini. Così fare di una mosca un elefante, un'espressione di origini latine (elaephantum ex musca facias), viene trovata nella lingua croata come equivalente totale, anche con alcune varianti (pulce, zanzara), mentre il ceco prende la variante evocata dalla Bibbia ('fare di una zanzara un cammello') (Opašić 2014, 11). Invece, tignola dei libri viene trovato in tutte e tre le lingue come equivalente totale (un chiaro calco dall'inglese e dal tedesco 'bookworm', 'bucherwurm'). Infine, cercare le pulci a qualcuno in croato lo fa una persona pignola (cro. biti picajzla 'essere una cimice', che in italiano ha un altro significato 'avaro, grezzo, vile'). Mentre in tutte e tre le lingue esiste la variante con il pelo (cercare/trovare il pelo nell'uovo - cec. 'cercare il pelo in tutto'). Infine, una persona che ha una scrittura che sembra zampe di mosche è una persona con una calligrafia estremamente brutta e mentre nell'italiano troviamo di nuovo un insetto, nelle due lingue slave abbiamo le varianti (oltre ad altre pisati lijevom nogom, škrabopis) con altri animali cr. švrakopis, pisati kao svraka 'scrivere come una gazza', mentre in ceco 'scriviamo come i porci' škrabe jako prase.

3. Stati d'animo

k) Stanchezza (lentezza) vs. azione (velocità) - Quando qualcuno si sente infelice o avvilito (insultato) o giù di corda, in italiano si sente come una cimice calpestata (già piccola e insignificante e per di più calpestata) un'immagine mentale diversa da quella croata e ceca ('come una gallina sotto la pioggia'), mentre in croato troviamo anche un volgarismo 'come un piccione cagato' ${ }^{9}$. Se pensiamo invece

${ }^{9}$ In croato per il significato di essere soltanto stanco si dice umoran kao pas 'stanco come un cane' ili osjećati se/izgledati kao prebijena mačka 'sentirsi come un gatto malmenato'. 
ad una persona che fa le cose lentamente in italiano di nuovo troviamo la variante con un insetto andare a passi di formica, accanto alle varianti con altri animali come la tartaruga o la lumaca (presente anche in croato). In ceco, invece, l'espressione presenta il gambero. Gli equivalenti parziali in tutte e tre le lingue contengono un animale che attraverso il suo comportamento (il camminare) stimola un'immagine mentale diretta. Interessante l'espressione cadere come le mosche 'morire in gran numero', equivalente totale in tutte e tre le lingue che possibilmente è stato motivato dallo spruzzare gli insetticidi sulle mosche che cadono e muoiono in gran fretta, mentre le interpretazioni inglesi alludono alla fragilità della mosca quando entra in casa. Questo significa che dovrebbe essere stato prodotto soltanto negli ultimi 100 anni e diffuso e tradotto in diverse lingue. L'immagine mentale che è direttamente legata alla velocità fisica è presente anche nell'espressione sparpagliarsi come uno sciame d'api 'fuggire velocemente (in gran numero) senza alcuna direzione'. Gli equivalenti diversi in croato sono composti da animali, 'piccoli granchi', mentre in ceco 'si sparpagliano in tutte le direzioni' senza alcun riferimento ad animali.

I) Rabbia/dolore - Quando qualcuno si arrabbia in italiano può far vedere le lucciole a qualcuno, l'espressione idiomatica con una chiara immagine mentale in cui qualcuno viene picchiato, esistente, però, soltanto in italiano. II significato è altamente diverso senza il verbo fare, perché include un acutissimo dolore che può non essere causato dalla rabbia. Gli equivalenti diversi in ceco ('vedere tutti i santi' - equiv. anche in croato) e in croato ((far) vedere tutte le stelle - equiv. anche in italiano) sono comunque senza un insetto. Dall'altra parte una persona che si offende in italiano piglia il grillo (cr. namusiti se, cec. nosit hlavu (nos) vzhuru), il che di nuovo non coincide con le altre lingue, ma neanche l'espressione ceca e quella croata coincidono formalmente.

4. Significativo vs. irrilevante - Esistono espressioni o modi di dire in italiano che includono nel significato il valore o poca rilevanza di qualcuno e vengono motivati dagli insetti. Di nuovo, le espressioni permeate di significati negativi sono molto più presenti. Così qualcuno che fa un lavoro inutile va $a$ caccia di grilli, un'espressione che non ha alcun equivalente 
nelle due lingue slave. Non valere una cicala è la persona che non vale niente ed ha diversi equivalenti in croato e in ceco però senza l'insetto (cr. 'non valere una fava baccata', cec. nema ani za groš ceny - simile in italiano). ${ }^{10}$ Con l'avere il sangue di piattola non si descrive soltanto una persona insignificante, ma anche vile. In croato non esiste un equivalente idiomatico, mentre in ceco il significato è stato parzialmente mantenuto nell'espressione 'essere una piattola'. II proverbio anche le pulci hanno la tosse ${ }^{11} \mathrm{di}$ etimologia napoletana trova un suo equivalente diverso in croato nel prestito pik-zibner con il significato di nuovo di una persona insignificante, che in ceco invece ha nuovamente una forma generale 'una persona è nulla'. Infine, se parliamo dei significati positivi vediamo che esiste un modo di dire con l'insetto mosca che chiaramente e trasparentemente esprime l'importanza e la rarità di una persona. Essere una mosca bianca è una espressione diversa dal croato e dal ceco che usano una variante calcata dal latino rara avis 'cornacchia bianca'.

5. Il mangiare - Nelle lingue slave esistono espressioni riguardanti la sfera del cibo, aventi significati poco costruiti e che utilizzano diversi uccelli invece degli insetti (cro. jesti kao ptica, kao pilić, kao vrapčić; cec. jist jako vrabec), mentre in italiano di nuovo troviamo la costruzione con un insetto mangiare quanto una formica.

Poche altre espressioni che usano l'insetto e non si riferiscono agli stati psicologici, a caratteristiche personali oppure a stati d'animo sono non sentir volar una mosca, un equivalente totale con il croato (mentre in ceco è completamente diverso) e andare a sentir cantare i grilli, un eufemismo di 'morire' che contiene un equivalente diverso nelle lingue slave 'andare alla verità di Dio'.

\subsection{Analisi statistica}

a) Delle 72 espressioni idiomatiche trovate in italiano, soltanto 7 espressioni hanno equivalenti totali (uguali componenti frasali + uguale significato) in tutte e tre le

\footnotetext{
${ }^{10}$ Così in italiano abbiamo anche non valere una cicca / un cavolo / una pera cotta / un fico secco / un soldo bucato.

${ }^{11}$ Il significato del detto italiano è diverso se si usa con i bambini. Le pulci sono talmente piccole che nessuno potrebbe sentire la loro voce e le loro opinioni (inesperte) suonano fuori luogo come l'improvviso colpo di tosse di una pulce. E' un modo scherzoso di dire 'lascia parlare i grandi' (vesuviolive.it).
} 
lingue. Qui si potrebbero aggiungere altre due espressioni che si potrebbero considerare equivalenti totali (anche se il verbo è quello che cambia minimamente). Così arriviamo a nove equivalenti totali:

Tabella 1. Equivalenti totali nelle tre lingue

\begin{tabular}{lll}
\hline ITALIANO & CROATO & CECO \\
\hline $\begin{array}{l}\text { operoso come un'ape } \\
\text { avere (sentire) le farfalle } \\
\text { nello stomaco }\end{array}$ & $\begin{array}{l}\text { marljiv kao pčela } \\
\text { imati leptiriće u } \\
\text { trbuhu }\end{array}$ & $\begin{array}{l}\text { pilný jako včela } \\
\text { mít motýly (motýlky) } \\
\text { v břiše }\end{array}$ \\
$\begin{array}{l}\text { formica } \\
\text { lavorare come una formica }\end{array}$ & $\begin{array}{l}\text { raditi kao mrav } \\
\text { pilný jako mravenec } \\
\text { dělat (pracovat) jako } \\
\text { mravenec }\end{array}$ \\
$\begin{array}{l}\text { cadere (morire) come le } \\
\text { mosche }\end{array}$ & $\begin{array}{l}\text { umirati (padati) } \\
\text { kao muhe }\end{array}$ & $\begin{array}{l}\text { padat (umírat) jako } \\
\text { mouchy }\end{array}$ \\
$\begin{array}{l}\text { noioso come una mosca } \\
\text { knjiški moljac } \\
\text { essere parsimonol } \\
\text { attivo come una formica; } \\
\text { essere una formica; fare la } \\
\text { formica }\end{array}$ & kosadan kao muha mrav; raditi & $\begin{array}{l}\text { kao mrav } \\
\text { moucha }\end{array}$ \\
$\begin{array}{l}\text { kumerosi come formiche } \\
\text { numlat (pracovat) jako } \\
\text { mravenec }\end{array}$ \\
\hline
\end{tabular}

b) In 6 casi l'espressione italiana è equivalente soltanto al croato e in 2 casi soltanto al ceco. Questo ci porta al numero di 17 equivalenti totali con l'italiano. Per gli equivalenti parziali con l'italiano esiste soltanto una espressione croata: avere grilli per la testa/ che è identica in ceco/ imati mušice/bubice u glavi. Mentre un esempio in ceco esiste come equivalente parziale con l'italiano aggirarsi come una mosca senza capo (la variante ceca non comprende 'la mosca')/juriti kao muha bez glave.

Fino a qui possiamo concludere che ci sono più equivalenti totali tra il croato e l'italiano (15) che tra l' italiano e il ceco (11).

c) In croato la disuguaglianza totale è visibile in 48 casi con l'italiano, mentre tra il ceco e l'italiano esistono 52 espressioni diverse. In tutto il corpus degli equivalenti diversi (totale 55) dall'italiano alle altre due lingue, notiamo che tra la lingua croata e quella ceca esistono invece 24 equivalenti totali. 
Tabella 2. Equivalenti diversi in almeno 2 lingue.

\begin{tabular}{|c|c|c|}
\hline ITALIANO & CROATO & CECO \\
\hline essere nero come un calabrone & biti crn kao Ciganin & černý jako cikán \\
\hline noioso come un calabrone & dosadan kao muha & dotěrný jako moucha \\
\hline come una cimice calpestata & kao posrani (usrani) golub, kao pokisla kokoš & jako zmoklá slepice \\
\hline $\begin{array}{l}\text { girare intorno al lume come le } \\
\text { farfalle }\end{array}$ & $\begin{array}{l}\text { hodati kao po jajima, hodati na vrhovima } \\
\text { prstiju }\end{array}$ & chodit po špičkách \\
\hline (andare) a passi di formica & puževim korakom & jít (vléci se) hlemýždím (račím) krokem \\
\hline ammassare come una formica & skupljati kao hrčak što & být/bejt křeček \\
\hline avere il cervello di una formica & pileći (kokošji, ptičji) mozak & mít slepičí rozum \\
\hline mangiare quanto una formica & jesti kao ptica (ptičica, vrabac, vrapčić) & jíst jako vrabec (vrabeček) \\
\hline cervello di grillo & pileći (kokošji, ptičji) mozak & pileći (kokošji, ptičji) mozak \\
\hline cuore di grillo & plašljiv kao zec; zečje srce & $\begin{array}{l}\text { mít zaječí srdce; bázlivý (vyplašený, } \\
\text { vylekaný) jako zajíc (králík) }\end{array}$ \\
\hline indovinala grillo! & vrag bi ga znao! & čert ví \\
\hline mangia come un grillo/ formica & jesti kao ptica (ptičica, vrabac, vrapčić) & jíst jako vrabec (vrabeček) \\
\hline $\begin{array}{l}\text { non cavar un grillo (un ragno) da un } \\
\text { buco }\end{array}$ & ništa mu ne ide za rukom & nejde od ruky někomu něco \\
\hline fare vedere a qc. le lucciole & $\begin{array}{l}\text { vidjeti sve zvijezde; vidjeti sve svetce na } \\
\text { nebu }\end{array}$ & vidět všechny svaté \\
\hline essere come una mosca nel latte & bosti u oči & bít (moc) do očí/vočí \\
\hline far saltare la mosca al naso a qc. & ići na živce kome & lézt (jít) na nervy někomu \\
\hline fare la mosca cocchiera & $\begin{array}{l}\text { šepuriti se (napuhavati se i sl.) kao puran } \\
\text { (paun) }\end{array}$ & jako krocan; naparovat se jako krocan \\
\hline mosca bianca & bijela vrana & být (mezi někým/někde) bílá vrána \\
\hline numerosi come mosche & kao mrava <koga > & je někoho <tam> jako mravenců \\
\hline magro come un moscerino & suh kao komarac; mršav kao glista & $\begin{array}{l}\text { slabý (hubený) jako komár; být hubený } \\
\text { jako žǐžala }\end{array}$ \\
\hline $\begin{array}{l}\text { stare zitto (tacere) come un } \\
\text { pidocchio }\end{array}$ & šutjeti kao kurva & $\begin{array}{l}\text { mlčet (držet hubu) jako kurva; být/bejt } \\
\text { huba líná }\end{array}$ \\
\hline noioso come una pulce & dosadan kao muha & dotěrný jako moucha \\
\hline essere noioso come una vespa & dosadan kao muha & dotěrný jako moucha \\
\hline pungere come una vespa & $\begin{array}{l}\text { imati oštar jezik; nemati dlake na jeziku; } \\
\text { zmijski jezik }\end{array}$ & $\begin{array}{l}\text { mít ostrý jazyk; mít jazyk jako břitva; } \\
\text { mít jazyk z hadích ocásků (spletený) }\end{array}$ \\
\hline grattare il corpo/pancia alla cicala & natjerati (navesti) nekoga da propjeva & donutit ptáčka zpívat \\
\hline andare a sentir cantare i grilli & otići Bogu na istinu (račun) & $\begin{array}{l}\text { odejít k Abrahamu; odejít na pravdu } \\
\text { boží }\end{array}$ \\
\hline $\begin{array}{l}\text { saltare <su }>\text { come punto da una } \\
\text { vespa }\end{array}$ & skočiti (odskočiti) kao ofuren/ oparen & vyskočit jako když ho vosa bodne \\
\hline essere come una zanzara & dosadan kao muha & dotěrný jako moucha \\
\hline $\begin{array}{l}\text { fastidioso (noioso) come una } \\
\text { zanzara }\end{array}$ & dosadan kao muha & dotěrný jako moucha \\
\hline
\end{tabular}

Le differenze si vedono specialmente nelle varianti metaforiche di 'essere fastidioso' che in italiano sono varie: come una zanzara, calabrone, vespa, pulce, mosca, mentre nel ceco e nel croato sono sempre le stesse varianti come una mosca/una pulce. Come si vede dalla tabella, spesso le varianti italiane contengono un insetto, mentre quelle croate e ceche uccelli o altri animali (vrabac, ptica, kokoš, hrčak, vrana, rak). Ci sono anche varianti che in italiano contengono un insetto, mentre le espressioni croate e ceche sono più generiche. 
d) Equivalenza zero

Fino a questo punto sono state citate le equivalenze unidirezionali che vanno dall'italiano alle altre due lingue (ceco e croato). Cambiando, invece, la prospettiva e cercando le espressioni croate in contrasto con quelle italiane si scopre che alcune sono specificamente croate (non presenti né in italiano, né in ceco) pala muha na medvjeda ossia 'una cosa da niente, insignificante'; kolju se kao žuti mravi 'si ammazzano fino alla fine'; otkini komarcu nogu odnio si mu život; una variante di 'fare di una mosca un elefante' praviti od komarca magarca (praviti od buhe slona); leglo buha 'prljavo mjesto'. Alcuni insetti non erano presenti in italiano, mentre in croato e in ceco formano espressioni idiomatiche come: ubiti jednim udarcem dvije muhe/prendere due piccioni con una fava/ zabit dvě mouchy jednou ranou; lijen kao trut/ 'pigro come un fuco'/ líní lidští trubci; kao vreća buha (per un fanciullo vivace)/ snáze uhlídás pytel blech než, mentre alcuni esistono soltanto in croato buba na vratu 'invadente come un parassita', lijena buba 'essere pigri'.

\section{Conclusione}

Bartminski $(2007,96)^{12}$ ha constatato che gli stereotipi generati dagli esseri umani su animali in generale appartengono (così come per le piante) agli stereotipi più antichi e possiedono per questo un significato culturale specifico. L'uomo è stato da sempre a diretto contatto con gli insetti, anche quando si è trasferito in zone urbane. Questo contatto ha fatto sì che l'uomo fosse motivato a creare espressioni idiomatiche che non sembrano scomparire né essere meno produttive. A differenza dalle espressioni riguardanti il mondo animale, che sembrano abbastanza universali e spesso combaciano in tutte e tre le lingue (cervello di gallina, fare il pavone, lingua di serpente), per quanto riguarda gli insetti si nota una specificità culturale (specialmente per l'italiano). In questo modo le varianti con gli animali hanno anche una variante con l'insetto (in italiano) cervello di formica/grillo, pidocchio rifatto, pungere come una vespa.

Quantitativamente (avendo fatto l'analisi contrastiva unidirezionale dal corpus italiano a quello croato e ceco) possiamo concludere che la lingua italiana utilizza due volte di più le espressioni idiomatiche con gli insetti rispetto alla lingua croata (72 vs. 34 ) e 2,5 di più della lingua ceca (72 vs. 28), ossia presenta una produzione molto alta. Inoltre, ci sono degli insetti che in confronto alle lingue slave non vengono riportati nelle espressioni idiomatiche o vengono riportati molto raramente,

${ }^{12}$ In Vidović Bolt (2014: 7). 
come per esempio il grillo, la cicala, il calabrone, la lucciola, la vespa. Tenendo conto della posizione geografica e delle specificità climatiche può anche essere spiegata la mancanza di questi insetti nelle espressioni idiomatiche. Per esempio le cicale ed i grilli sono molto rari nelle zone a nord e a sud del $55^{\circ}$ parallelo. Così per il ceco è anche evidente che le espressioni idiomatiche non siano motivate da insetti che in questa zona sono rari o non esistono affatto. Non si può affermare lo stesso nel caso del croato, dove l'habitat è simile a quello italiano. In questo senso si può dire che la produzione delle espressioni idiomatiche con gli insetti menzionati è culturo-specifica della lingua italiana.

Da un punto di vista semantico, l'analisi ha assolutamente confermato che la lingua italiana (in confronto alle altre lingue) abbonda di espressioni idiomatiche, che con la componente dell'insetto rendono benissimo alcune caratteristiche psicologiche dell'uomo, i suoi difetti o il suo stato d'animo. Quelle più produttive sembrano cogliere il concetto ed il significato di noia o di invadenza. Anche se l'italiano usa insetti diversi (eccetto la mosca), il croato ed il ceco sono altrettanto produttivi (con gli stessi insetti). Una situazione simile viene trovata nel concetto di laboriosità. Le espressioni con l'ape e la formica sono produttive in tutte e tre le lingue, mentre la sua variante negativa, la pigrizia, è assolutamente specifica nell'uso degli insetti nella lingua italiana (guardare le mosche che volano, pigliar mosche, fare come la cicala). La lingua italiana in questo caso sembra essere molto produttiva nell'esprimere la poca laboriosità dell'uomo. La rappresentazione del concetto di paura con l'insetto in italiano è più forte nelle espressioni, in contrasto con le altre due lingue che generalmente usano altri animali (cervo, coniglio, cagnolino).

Infine, ciò che mette in evidenza la similarità tra le tre lingue nelle espressioni idiomatiche con gli insetti sono i calcoli quantitativi di equivalenza. Delle 72 espressioni idiomatiche trovate in italiano, soltanto 9 espressioni (il 12\%) sono equivalenti totali in tutte e tre le lingue. Separando le lingue vediamo un'affiliazione più stretta di equivalenti totali tra le lingue slave (24), piuttosto che tra il croato e I'italiano (15) e tra il ceco e l'italiano (11). Ciò sta a significare che, come ipotizzato, la più forte equivalenza esiste tra le lingue slave, segue l'equivalenza tra il croato e l'italiano ed infine tra il ceco e l'italiano. Ciò viene confermato anche dalle disuguaglianze totali che sono molto più presenti tra l'italiano e il ceco (di tutti gli equivalenti diversi, 55, 53 sono con il ceco) che tra l'italiano ed il croato (di tutti gli equivalenti diversi, 48 sono con il croato). 
Questi risultati ci hanno confermato quanto l'affiliazione storicoculturale e linguistica possa essere forte nella produzione e nella conservazione di espressioni idiomatiche. Le lingue slave (croato e ceco), anche se geograficamente e storicamente più lontane, hanno conservato le loro specificità idiomatiche, anche dopo almeno un millennio di distanza linguistica. D'altra parte l'italiano, essendo nell'ultimo millennio in stretto rapporto con il croato, ha sicuramente lasciato più repertorio idiomatico nel croato che nel ceco (ciò è evidente proprio nella specificità di questo campo idiomatico nel quale è presente la componente degli insetti; oltre alle scritture bibliche è riuscito ad essere trasmesso anche tramite contatti diretti e calchi linguistici).

\section{Bibliografia:}

Anić, Vladimir. Veliki rječnik hrvatskoga jezika. Zagreb: Novi Liber, 2003. Barčot, Branka. "Antropomorfizam i zoomorfizam u hrvatskim, ruskim i njemačkim zoonimskim frazemima", in Philological Studies (1857-6060) 2 (2014): 481-496.

Bunk, Ana e Maja Opašić. "Prilog kontrastivnoj analizi frazema sa zoonimskom sastavnicom u hrvatskome i češkome jeziku", in Rasprave: Časopis Instituta za hrvatski jezik i jezikoslovlje. Vol. 36, No.2. (2011): 237-250.

Casadei, Federica. Metafore ed espressioni idiomatiche. Roma: Bulzoni , 1996.

Deanović, Mirko e Josip Jernej. Hrvatsko-talijanski rječnik. Zagreb: Školska knjiga, 1994.

Vocabolario italiano-croato. Zagreb: Školska knjiga, 2012.

Fink Arsovski, Željka. Hrvatsko-slavenski rječnik poredbenih frazema. Zagreb: Knjigra, 2006.

---------Hrvatsko-romansko-germanski rječnik poredbenih frazema. Zagreb: Knjigra, 2016.

Holuša, Jaroslav et all. "Southern Cricket Eumodicogryllus bordigalensis (Orthoptera: Gryllidae) in the Czech Republic: new records and notes on the biology and stridulation", in Polish Journal of Entomology. P O L I S H VOL. 76 (2007): 47-55.

Jelaska, Zrinka. "Animalistički frazemi biblijskoga podrijetla u hrvatskomu i drugim slavenskim jezicima", in Animalistički frazemi u slavenskim jezicima. Zagreb: Ff Press (2014): 1-22.

Jernej, Josip. "Bilješke oko porijekla naše frazeologije", in Suvremena lingvistika. Vol. 41-42, No. 1-2 (1996): 265-269. 
Menac, Antica, Željka Fink Arsovskie Radomira Venturin. Hrvatski frazeološki rječnik. Zagreb: Naklada Ljevak, 2014.

Menac, Antica, Dubravka Sesar e Renata Kuchar. Hrvatsko-češkoslovački frazeološki rječnik: s indeksom čeških i slovačkih frazema. Zavod za lingvistiku Filozofskog fakulteta Sveučilišta, Zagreb, Čakovec: "Zrinski", 1998.

Menac, Antica e Zorica Vučetić. Hrvatsko-talijanski frazeološki rječnik/ Vocabolario fraseologico croato-italiano. Zagreb: Zavod za lingvistiku Filozofskog fakulteta Sveučilišta, 1995.

Opašić, Maja. "Biblijski onimi u hrvatskome jeziku”, in Folia onomastica croatica 23, Rijeka, (2014): 185-208.

. "Općeeuropski frazemi hrvatskoga jezika u Rječniku stranih riječi Bratoljuba Klaića”, in Časopis Instituta za hrvatski jezik $i$ jezikoslovlje 40/2, Rijeka, (2014): 411-434.

"Zoonimske sastavnice u biblizmima hrvatskoga i pojedinih jezika", in Životinje u frazeološkom ruhu. Rijeka, 2014. 1-14.

Turk, Marija e Nina Spicijarić Paškvan. “Kontrastivna raščlamba kao postupak otkrivanja podrijetla frazema (na primjeru frazema sa zoonimskom sastavnicom)", in Životinje u frazeološkom ruhu. Sveučilište u Rijeci, 2014. 1-14.

Vidović Bolt, Ivana. "Životinja kao (ne)inteligentan čovjekov prijatelj”, in Životinje u frazeološkom ruhu. Zagreb: FF press, 2014. 1-12.

Zingarelli, Nicola. Lo Zingarelli 1996: Vocabolario della lingua italiana, dodicesima edizione. Zanichelli 1996.

\section{Sitografia:}

Baza frazema hrvatskoga jezika: http://frazemi.ihjj.hr/, 3.3.2018.

Dizionario dei Modi di Dire: http://dizionari.corriere.it/dizionario-modidi-dire/, 4.3.2018.

Dizionari di Lingua Italiana: http://www.sapere.it/sapere/dizionari.html, 15.3.2018.

Treccani Vocabolario on line: http://www.treccani.it/vocabolario/, 11.2.2018.

Grande Dizionario Hoepli Italiano di Gabrielli Aldo Dizionario della Lingua Italiana:

http://www.grandidizionari.it/Dizionario_Italiano, 20.1.2018.

De Agostini enciclopedia: http://www.sapere.it/sapere/dizionari.html, 6.12.2017.

Glossario delle frasi fatte: https://it.wikipedia.org/wiki/Glossario_delle_frasi_fatte, 3.12.2017.

Dizionario italiano Olivetti: http://www.dizionario.italiano.it, 10.11.2017. 
Hrvatski jezični portal: http://hjp.znanje.hr/, 5.3.2018.

Slovník spisovného jazyka českého: http://ssjc.ujc.cas.cz/, 9.3.2018.

DEBDict: https://deb.fi.muni.cz:8005/debdict/, 15.3.2018.

\section{ENTOMOLOGIJA U TALIJANSKIM FRAZEMIMA I NJIHOVI HRVATSKI I ČEŠKI EKVIVALENTI}

U ovom radu kroz kontrastivnu analizu istražujemo koliko i kako se izgled, ponašanje i obilježja pojedinih kukaca (koji se načelno smatraju univerzalnima) unutar frazema razlikuju u tri jezika (talijanski, hrvatski i češki). Počevši od talijanskih frazema s entomološkom sastavnicom (17 različitih kukaca) pronalazimo moguće ekvivalente u hrvatskom i češkom jeziku.

S jedne strane možemo pretpostaviti da će hrvatski i češki frazemi imati veću učestalost apsolutnih ekvivalenata s obzirom da dijele zajednički prajezik, ali i s obzirom na zajedničko povijesno kulturno nasljeđe (npr. biblijska terminologija). S druge strane, pretpostavljamo da su direktni hrvatsko-talijanski jezični i kulturni dodiri doveli do više afilijacije talijanskog jezika s hrvatskim, nego li s češkim.

Slijedeći metodološke korake kontrastivne analize opisali smo sličnosti i različitosti frazema u sva tri jezika (pronalazeći apsolutne, parcijalne, različite formalne i semantičke ekvivalente, i one $s$ nultom ekvivalencijom) te smo kroz konceptualnu analizu frazema objasnili izbor pojedinog jezika da se koristi obilježjima kukaca za pojedina psihološka stanja i radnje.

Od 72 talijanskih frazema sa sastavnicom kukaca samo je $12 \%$ (9) frazema apsolutnih ekvivalenata u sva tri jezika. No, ukoliko razdvojimo jezike pronalazimo najviše sličnosti u slavenskim jezicima (čak 24 apsolutna ekvivalenta), zatim među talijanskim i hrvatskim jezikom (15 apsolutnih ekvivalenata), te nešto manje među talijanskim i češkim jezikom (11 apsolutnih ekvivalenata). I konceptualna analiza potvrđuje najjaču afilijaciju hrvatskog i češkog jezika.

Različiti formalni i semantički ekvivalenti podržavaju isti zaključak. Slavenski jezici (hrvatski i češki), iako povijesno i geografski udaljeniji, u jezičnom su smislu često zadržavali zajedničke frazemske specifičnosti. $S$ druge strane, budući da je talijanski jezik (odnosno romanski dijalekti) već jedno tisućljeće u bliskom doticaju s hrvatskim jezikom (odnosno njegovim dijalektima), za razliku od češkog, isti se doticaj sada vidi i u hrvatskim frazemima koji su ipak sličniji talijanskom u odnosu na češke.

Ključne riječi: frazemi, kontrastivna analiza, konceptualna analiza, kukci, ekvivalenti, talijanski, hrvatski, češki. 